\title{
Maternal mediation as perceived privacy invasion for adolescent internet use
}

\section{Yih-Lan Liu Taiwan, Institute of Education/ Center for Teacher Education, National Chiao Tung University,}

\section{Introduction}

Internet use has become a daily activity for most of people nowadays, not excluded adolescents. Although the internet has provided adolescent opportunities for leaning, social interaction, entertainment, and a communication tool with families, the internet may connote negative aspects such as exposure to online risk and internet addiction

Parents employ strategies to regulate children's behavior to protect them from excessive internet use. However, as children enter adolescence, they will see an expanding range of issue as falling outside parents' legitimate right to know. They begin to fortify privacy boundaries with parents. Issues of privacy invasion may arise when adolescent have the feeling that parents desire or acquire information that they consider to be personal. This privacy invasion results from the mismatch between the adolescent's belief that some issues should be private and parental convictions that they have a right to know or to intervene (Hawk et all., 2013). To restore privacy boundary, adolescents start to keep secrets or to develop defensive behaviors (Ledbetter et al., 2010), which may ultimately cause psychopathology or other behavioral problems (Dietvorst et al., 2007).

By using a two-year data, this study hypothesized that maternal mediation of adolescent internet use might be perceived as the privacy invasion by adolescents, which further provoke defensive behaviors (e.g disclose the information of internet use), and such defensive behaviors would increase the risk of internet use such as exposure to risk of internet use such as
online risk and internet addiction.

\section{Methods}

Participants: 678 adolescents and their mothers from junior high and senior high schools in northern, central, and southern Taiwan (880 7th graders; 645 10th graders).

Procedure: In the first year, invitation letters were sent to schools. After obtaining parents' consent forms, adolescents and their mothers filled out questionnaires at home, and the questionnaires were collected by school teachers. The second-year data were collected by the similar procedure.

\section{Measures}

1.Maternal medication strategies (mothers' reports at T1) : active medication ( $\alpha=.88$ ); restriction medication $(\alpha=.80)$; co-using mediation ( $\alpha=.91$ for adolescents' version) 2. Perceived privacy invasion(adolescent report at T2): perceptions of privacy invasion for internet use, $\alpha=.87$.

3. Adolescent defensive behaviors (adolescent report at T2): include secrecy defense, mediated defense, and avoidance defense, $\alpha=.91$

4. Exposure to internet risk (adolescent report atT1 \& T2): the experiences of exposure to inappropriate websites, disclosure of personal information to strangers...etc., $\alpha=$ 65 at $\mathrm{T} 1$ and .69 at $\mathrm{T} 2$.

5. Internet addiction(adolescent report atT1 \& T2) : the degrees of possibility not to become internet addicted, $\alpha=.94$ at $\mathrm{T} 1$ and .94 at $\mathrm{T} 2$.

\section{Restults}

Table 1 displays descriptive analyses including mean, standard deviations, and correlations among variables. To measure the proposed hypotheses, we used Structure equation modeling (SEM) analysis to examine the proposed mediation model. the result indicated a good model fit, too, $\chi 2$ $(988)=2198.15, \mathrm{CFI}=.92, \mathrm{TLI}=.91$, RMSEA $=.043(90 \% \mathrm{Cl}=0.41-.046)$, SRMR $=.054$, with factor loadings of each indictor ranged from .32 .87 (all ps <. 01). As indicated in Figure 1, maternal restrictive mediation at $\mathrm{T} 1$ was positively correlated with adolescent perceived privacy invasion $(\beta=.18, p<.01)$, but maternal active and co-use mediation showed no relations. Perceived privacy invasion at T2 was positively correlated with privacy defense at T2 $(\beta=.66, p<.001)$.
Privacy defense was positively correlated with exposure to online risk at T2 $(\beta=.14, p$ $<.01)$ and internet addiction at T2 $(\beta=.22, p$ $<.001)$.

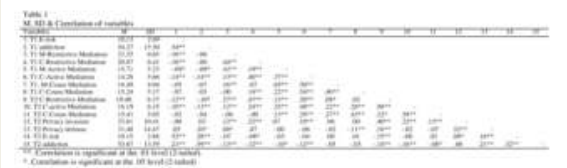

\section{Conclusions}

Compared to co-using and active maternal mediation, restrictive mediation was more likely to be seen as the invasion of privacy by adolescents. When adolescents perceived the privacy boundaries were violated, they tend to use defensive behaviors by disclosing information about internet use, and this would increase the risk of online risk and internet addiction

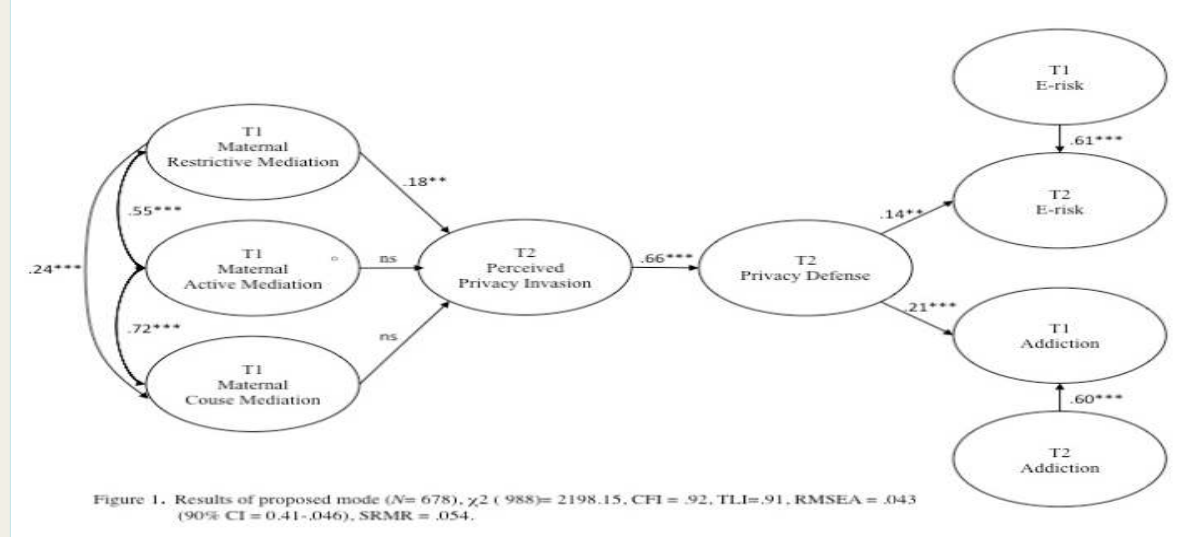

This could be a place for your sources. 